\title{
Generalized beam quality factor of aberrated truncated Gaussian laser beams
}

\author{
Cosmas Mafusire ${ }^{1,2}$ and Andrew Forbes ${ }^{1,2, *}$ \\ ${ }^{1}$ Council for Scientific and Industrial Research National Laser Centre, P.O. Box 395, Pretoria, South Africa \\ ${ }^{2}$ School of Physics, University of KwaZulu-Natal, Private Bag X54001, Durban 4000, South Africa \\ ${ }^{*}$ Corresponding author: aforbes1@csir.co.za
}

Received March 8, 2011; revised May 9, 2011; accepted May 9, 2011;

posted May 9, 2011 (Doc. ID 143785); published June 9, 2011

We outline a theory for the calculation of the beam quality factor of an aberrated laser beam. We provide closedform equations that show that the beam quality factor of an aberrated Gaussian beam depends on all primary aberrations except tilt, defocus, and $x$-astigmatism. The model is verified experimentally by implementing aberrations as digital holograms in the laboratory. (c) 2011 Optical Society of America

OCIS codes: $\quad 140.3295,080.1005,120.5050$.

\section{INTRODUCTION}

The laser beam quality factor $\left(M^{2}\right)$ is a useful parameter for describing laser beam propagation through linear optical systems and can be used directly to calculate how tightly a laser beam may focus or how fast it diverges during propagation [1,2]. Since this factor does not change when propagating through media with a quadratic refractive index profile, a change in the beam quality factor can be used to infer the presence of aberrations and thereby study the media of interest. For these reasons, among others, research into the beam quality factor of Gaussian beams has generated much interest in the laser community [1,2]. However, despite the large body of work on the subject of laser beam propagation, to date very little work has considered how the change in laser beam quality is related to aberrations.

Optical aberrations have been a subject of interest for some time now [3-7]. They have found use in the fields of imaging [3,4], ophthalmology [5], and atmospheric turbulence [4,7]. Their use in laser beams has mostly been limited to the aberrations' impact on the laser field during propagation [8-10]. Research on their impact on individual beam parameters such as the beam quality factor, beam size, waist size, and waist location has been limited at best. It follows that the beam quality factor is a measure of a real beam's deviation from the "ideal" Gaussian mode, both in amplitude and in phase $[\underline{1}, \underline{2}, \underline{11}]$. The latter point suggests that, when aberrations are present, a concomitant decrease in the beam quality factor should be noted. Siegman and coworker showed both theoretically [12] and experimentally [13] how a lens aberrated by spherical aberration can degrade the quality of a laser beam of any size and that, beyond a certain size, the $M^{2}$ factor increases sharply. This model was extended to a tensor formulation to include all primary Zernike coefficients in the characterization not only of $M^{2}$ but also of divergence and curvature but was not tested experimentally [14]. An attempt to use the model in experiments has not been successful as the model is deemed to be "so mathematical, that it might not be appropriate to apply in practical applications" [15]. It is probably because in [14] the beam quality factor is presented in the form of a tensor, $J$, which is of little practical value but very important in theoretical analysis of laser beams.

In this paper we propose a model for the impact of aberrations on the laser beam quality factor. Our model, based on the moments concept, is practical to use and easy to demonstrate in the laboratory. In particular we propose a formulation for describing the impact of aberrations on the laser beam quality factor of Gaussian beams. We will show that this formalism leads to a closed-form solution in terms of aberrations expressed in terms of the Zernike polynomial set and generalize the result to include Gaussian laser beams truncated by circular apertures of arbitrary size. The latter is important in such studies as any aberration description requires a very careful definition of the aperture over which the aberrations are defined. Our model predictions are in general agreement with those found by others, but moreover we present experimental validation of the results through the use of digital holograms written to a phase-only spatial light modulator. The accuracy of the agreement should prove this a useful tool for experimenters in the laboratory.

\section{THEORY}

The beam quality factor of a propagating field is invariant if no sources of aberrations are present in its path, and it may be calculated from the product of the near-field and far-field distributions of the beam [1]. Alternatively, the beam quality factor can be defined by the curvature removal method, in which a curvature fit is made to the wavefront at any position along the propagation path and subsequently subtracted to leave a beam "waist" from which the beam size (near field) and divergence (far field) are calculated, and the beam quality factor inferred. In the case where aberrations are present in the beam path, the beam quality factor is no longer invariant during propagation, and the latter method lends itself to computing the change in beam quality factor as a function of the propagation distance.

We can apply this method to a general complex amplitude transverse electric field, at some arbitrary propagation distance, given by 


$$
U(\rho, \theta)=\psi(\rho) \exp (i \phi(\rho, \theta))
$$

which carries power through some aperture of radius $a$, given by $P=a^{2} \int_{0}^{2 \pi} \int_{0}^{1} \psi^{2}(\rho, \theta) \rho \mathrm{d} \rho \mathrm{d} \theta$, where $\rho \in[0,1]$ and $\theta \in[0,2 \pi]$ with the radial term normalized to $a$. We can define a beam whose propagation for the two principal axes, $x$ and $y$, can be treated as separable problems. It can be shown that the beam quality factor using the curvature removal method is defined by (see Appendix $\underline{\text { A}}$ )

$$
\begin{aligned}
& M_{x}^{2}=\frac{\pi}{\lambda} \sqrt{\omega_{x}^{2} \theta_{x}^{2}-V_{x}^{2}}, \\
& M_{y}^{2}=\frac{\pi}{\lambda} \sqrt{\omega_{y}^{2} \theta_{y}^{2}-V_{y}^{2}},
\end{aligned}
$$

where the beam size, $\omega_{x}$, and the divergence, $\theta_{x}$, and their $y$-axis equivalents are given by

$$
\begin{aligned}
& \omega_{x}^{2}=\frac{4 a^{4}}{P} \int_{0}^{2 \pi} \int_{0}^{1} \psi^{2} \rho^{2} \cos ^{2} \theta \rho \mathrm{d} \rho \mathrm{d} \theta, \\
& \omega_{y}^{2}=\frac{4 a^{4}}{P} \int_{0}^{2 \pi} \int_{0}^{1} \psi^{2} \rho^{2} \sin ^{2} \theta \rho \mathrm{d} \rho \mathrm{d} \theta,
\end{aligned}
$$

$$
\begin{aligned}
\theta_{x}^{2}= & \frac{\lambda^{2}}{\pi^{2} P} \int_{0}^{2 \pi} \int_{0}^{1}\left(\cos ^{2} \theta\left(\frac{d \psi}{d \rho}\right)^{2}\right. \\
& \left.+\psi^{2}\left(\cos \theta \frac{\partial \phi}{\partial \rho}-\frac{\sin \theta}{\rho} \frac{\partial \phi}{\partial \theta}\right)^{2}\right) \rho \mathrm{d} \rho \mathrm{d} \theta-\frac{\lambda^{2} a^{2}}{\pi^{2} P^{2}} \\
& \times\left(\int_{0}^{2 \pi} \int_{0}^{1} \psi^{2}\left(\cos \theta \frac{\partial \phi}{\partial \rho}-\frac{\sin \theta}{\rho} \frac{\partial \phi}{\partial \theta}\right) \rho \mathrm{d} \rho \mathrm{d} \theta\right)^{2}+\theta_{e}^{2}
\end{aligned}
$$

$$
\begin{aligned}
\theta_{y}^{2}= & \frac{\lambda^{2}}{\pi^{2} P} \int_{0}^{2 \pi} \int_{0}^{1}\left(\sin ^{2} \theta\left(\frac{d \psi}{d \rho}\right)^{2}\right. \\
& \left.+\psi^{2}\left(\sin \theta \frac{\partial \phi}{\partial \rho}+\frac{\cos \theta}{\rho} \frac{\partial \phi}{\partial \theta}\right)^{2}\right) \rho \mathrm{d} \rho \mathrm{d} \theta-\frac{\lambda^{2} a^{2}}{\pi^{2} P^{2}} \\
& \times\left(\int_{0}^{2 \pi} \int_{0}^{1} \psi^{2}\left(\sin \theta \frac{\partial \phi}{\partial \rho}+\frac{\cos \theta}{\rho} \frac{\partial \phi}{\partial \theta}\right) \rho \mathrm{d} \rho \mathrm{d} \theta\right)^{2}+\theta_{e}^{2}
\end{aligned}
$$

The spatial-angular moment is given by

$$
\begin{aligned}
& V_{x}=\frac{2 \lambda a^{2}}{\pi P} \int_{0}^{2 \pi} \int_{0}^{1} \psi^{2} \rho \cos \theta\left(\cos \theta \frac{\partial \phi}{\partial \rho}-\frac{\sin \theta}{\rho} \frac{\partial \phi}{\partial \theta}\right) \rho \mathrm{d} \rho \mathrm{d} \theta, \\
& V_{y}=\frac{2 \lambda a^{2}}{\pi P} \int_{0}^{2 \pi} \int_{0}^{1} \psi^{2} \rho \sin \theta\left(\sin \theta \frac{\partial \phi}{\partial \rho}+\frac{\cos \theta}{\rho} \frac{\partial \phi}{\partial \theta}\right) \rho \mathrm{d} \rho \mathrm{d} \theta .
\end{aligned}
$$

Note that, in this formalism, we have introduced the truncation of the field (by some aperture) by limiting the integrals to finite size in the radial coordinate, which goes from 0 to 1 in the normalized state and from 0 to $a$ when not normalized. It is for this reason that the last term in Eqs. (4a) and (4b) appears: it accounts for the added divergence due to the finite aperture and approaches zero in the limit of a large aperture. It can be shown that it is given by [16]

$$
\theta_{e}^{2}=\frac{16 \lambda^{2}}{\pi^{2} P} \psi^{2}(1)
$$

It remains to introduce the aberrations to the field via an appropriate modulation of the phase function, $\phi(\rho, \theta)$. We choose to express the phase (or wavefront) as a linear combination of Zernike polynomials, orthogonal over the unit circle:

$$
\begin{aligned}
\phi(\rho, \theta)= & 2 \pi \sum_{n=0}^{m} A_{n 0} R_{n 0}(\rho) \\
& +2 \pi \sum_{n=1}^{\infty} \sum_{n=1}^{m} R_{n m}(\rho)\left[A_{n m} \cos m \theta+B_{n m} \sin m \theta\right]
\end{aligned}
$$

Here $R_{n m}(\rho)$ is the Zernike radial term and $n$ and $m$ (nonnegative integers) are the order and the ordinal numbers, respectively (the order is related to $m$ such that $m \leq n$ and $n-m$ is even). The Zernike odd and even coefficients in waves are represented by $A_{n m}$ and $B_{n m}$, respectively. The names, symbols and rms polynomials of the primary Zernike aberrations, which are of interest in this paper, are given in Table $\underline{1}$.

\section{ABERRATED GAUSSIAN BEAMS}

We select, by way of example only, the Gaussian field as defined by

$$
U(\rho, \theta)=\left(\frac{2 \gamma^{2}}{\pi a^{2}}\right)^{1 / 4} \exp \left(-\gamma^{2} \rho^{2}\right) \exp (i \phi(\rho, \theta)), \quad \rho \leq 1,
$$

where we have introduced a truncation parameter, $\gamma=\alpha / \omega$, to aid the analysis of aperture size on the properties of the beam. The consequence is that the expression in Eq. ( $\underline{8}$ ) is zero outside the aperture. Equation (ㅁ) can now be rewritten as

$$
\theta_{e}^{2}=\frac{32 \gamma^{2}}{\left(e^{2 \gamma^{2}}-1\right) \pi^{3}}\left(\frac{\lambda}{a}\right)^{2} .
$$

A graph of this function of the truncation parameter is shown in Fig. 1. It shows that, as the aperture size increases, the term approaches zero, which is referred to as the soft aperture case, and when $\gamma \geq 2$, we can ignore this term. Physically, it means that the beam is not experiencing diffraction effects

Table 1. Names, Symbols, and Polynomials of the Zernike Primary Aberration Coefficients

\begin{tabular}{cccc}
\hline$n$ & $m$ & Description and Symbol & Polynomial \\
\hline 0 & 0 & Piston, $A_{00}$ & 1 \\
1 & 1 odd & $y$-tilt, $B_{11}$ & $\sqrt{ } 2 \rho \sin \theta$ \\
& 1 even & $x$-tilt, $A_{11}$ & $\sqrt{ } 2 \rho \cos \theta$ \\
2 & 2 odd & $y$-astigmatism, $B_{22}$ & $\sqrt{ } 6 \rho^{2} \sin 2 \theta$ \\
& 0 & Defocus, $A_{20}$ & $\sqrt{ } 3\left(2 \rho^{2}-1\right)$ \\
& 2 even & $x$-astigmatism, $A_{22}$ & $\sqrt{ } 6 \rho^{2} \cos 2 \theta$ \\
3 & 3 odd & $y$-triangular astigmatism, $B_{33}$ & $\sqrt{ } 8 \rho^{3} \sin 3 \theta$ \\
& 1 odd & $y$-primary coma, $B_{31}$ & $\sqrt{8}\left(3 \rho^{3}-2 \rho\right) \sin \theta$ \\
& 1 even & $x$-primary coma, $A_{31}$ & $\sqrt{ } 8\left(3 \rho^{3}-2 \rho\right) \cos \theta$ \\
& 3 even & $x$-triangular astigmatism, $A_{33}$ & $\sqrt{ } 8 \rho^{3} \cos 3 \theta$ \\
4 & 0 & Spherical aberration, $A_{40}$ & $\sqrt{ } 5\left(6 \rho^{4}-6 \rho^{2}+1\right)$ \\
\hline
\end{tabular}


due to the aperture; the smaller this term, the greater diffraction it experiences and so the faster the beam diverges.

The beam quality factor of the Gaussian field is calculated using Eq. (2) after substituting for $\omega, \theta$, and $V$ as defined by Eqs. (3)-(5), respectively. The aberrations are defined in Eq. (7) and used as the expansion of the phase in Eq. (1). After much algebra, the results for the two principal planes are given by the $y$-astigmatism does. This is somewhat surprising considering that these two aberrations are similar in that each one is essentially a lens with two focal axes oriented orthogonally to one another. This is to say, they exhibit quadratic index behavior in axes orthogonal to each other. In $x$-astigmatism both focal axes coincide with the principal Cartesian axes, whereas with $y$-astigmatism the focal axes are at $45^{\circ}$ to the principal axes. This implies that, with respect to the Cartesian axes,

$$
\begin{aligned}
M_{x}^{4}= & \frac{1}{\pi\left[\exp \left(2 \gamma^{2}\right)-1\right] \gamma^{8}}\left\{2 4 \pi ^ { 3 } \left[B_{22}^{2}\left[\exp \left(2 \gamma^{2}\right)-1\right]\left[\exp \left(2 \gamma^{2}\right)-2 \gamma^{2}-1\right]^{2} \gamma^{4}+3\left(B _ { 3 1 } ^ { 2 } [ \operatorname { e x p } ( 2 \gamma ^ { 2 } ) - 1 ] [ \operatorname { e x p } ( 2 \gamma ^ { 2 } ) - 2 \gamma ^ { 2 } - 1 ] \left[\exp \left(2 \gamma^{2}\right)-1-2\left(\gamma^{4}\right.\right.\right.\right.\right. \\
& \left.\left.+\gamma^{2}\right)\right] \gamma^{2}+A_{31}^{2}\left[\exp \left(2 \gamma^{2}\right)-1-2 \gamma^{2}\right]\left[5 \exp \left(4 \gamma^{2}\right)+2 \gamma^{2}\left(\gamma^{2}+1\right)-2 \exp \left(2 \gamma^{2}\right)\left(9 \gamma^{4}+\gamma^{2}+5\right)+5\right] \gamma^{2} \\
& +2\left(B_{31} B_{33}+A_{31} A_{33}\right)\left[\exp \left(2 \gamma^{2}\right)-1\right]\left[\exp \left(2 \gamma^{2}\right)-1-2 \gamma^{2}\right]\left[\exp \left(2 \gamma^{2}\right)-2\left(\gamma^{4}+\gamma^{2}\right)-1\right] \gamma^{2}+\left[\exp \left(2 \gamma^{2}\right)-1\right]\left\{( B _ { 3 3 } ^ { 2 } + A _ { 3 3 } ^ { 2 } ) \left[\exp \left(2 \gamma^{2}\right)-1\right.\right. \\
& \left.\left.\left.\left.-2 \gamma^{2}\right]\left[\exp \left(2 \gamma^{2}\right)-1-2\left(\gamma^{4}+\gamma^{2}\right)\right] \gamma^{2}+20 A_{40}^{2}\left[2 \gamma^{2}\left(\gamma^{2}+2\right)+\exp \left(4 \gamma^{2}\right)-2 \exp \left(2 \gamma^{2}\right)\left(2 \gamma^{6}-\gamma^{4}+2 \gamma^{2}+1\right)+1\right]\right\}\right)\right] \\
& \left.+\left[\exp \left(2 \gamma^{2}\right)-1\right]\left[\exp \left(2 \gamma^{2}\right)-1-2 \gamma^{2}\right]\left\{\pi\left[\exp \left(2 \gamma^{2}\right)-1-2 \gamma^{2}\right]+32\right\} \gamma^{8}\right\}
\end{aligned}
$$

$$
\begin{aligned}
M_{y}^{4}= & \frac{1}{\pi\left[\exp \left(2 \gamma^{2}\right)-1\right] \gamma^{8}}\left\{2 4 \pi ^ { 3 } \left[B_{22}^{2}\left[\exp \left(2 \gamma^{2}\right)-1\right]\left[\exp \left(2 \gamma^{2}\right)-1-2 \gamma^{2}\right]^{2} \gamma^{4}+3\left(B _ { 3 1 } ^ { 2 } ( \operatorname { e x p } ( 2 \gamma ^ { 2 } ) - 1 - 2 \gamma ^ { 2 } ] \left[5 \exp \left(4 \gamma^{2}\right)+2 \gamma^{2}\left(\gamma^{2}+1\right)\right.\right.\right.\right. \\
& \left.-2 \exp \left(2 \gamma^{2}\right)\left(9 \gamma^{4}+\gamma^{2}+5\right)+5\right] \gamma^{2}+2 B_{31} B_{33}\left[\exp \left(2 \gamma^{2}\right)-1\right]\left\{\exp \left(2 \gamma^{2}\right)-1-2 \gamma^{2}\right\}\left[\exp \left(2 \gamma^{2}\right)-1-2\left(\gamma^{4}+\gamma^{2}\right)\right] \gamma^{2}+\left[\exp \left(2 \gamma^{2}\right)\right. \\
& -1]\left(\exp \left(4 \gamma^{2}\right)\left\{\left[B_{33}^{2}+\left(A_{31}-A_{33}\right)^{2}\right] \gamma^{2}+20 A_{40}^{2}\right\}+\gamma^{2}\left\{40 A_{40}^{2}\left(\gamma^{2}+2\right)+\left[B_{33}^{2}+\left(A_{31}-A_{33}\right)^{2}\right]\left[4 \gamma^{6}+6 \gamma^{4}+4 \gamma^{2}+1\right]\right\}\right. \\
& \left.\left.\left.-2 \exp \left(2 \gamma^{2}\right)\left\{\left[B_{33}^{2}+\left(A_{31}-A_{33}\right)^{2}\right]\left[\gamma^{3}+\gamma\right]^{2}+20 A_{40}^{2}\left(2 \gamma^{6}-\gamma^{4}+2 \gamma^{2}+1\right)\right]+20 A_{40}^{2}\right\}\right)\right]+\left[\exp \left(2 \gamma^{2}\right)-1\right]\left[\exp \left(2 \gamma^{2}\right)\right. \\
& \left.\left.-1-2 \gamma^{2}\right]\left[\pi\left(\exp \left(2 \gamma^{2}\right)-1-2 \gamma^{2}\right)+32\right] \gamma^{8}\right\},
\end{aligned}
$$

where we have made use of the orthogonality of the trigonometric functions as well as the radial component of the Zernike polynomials:

$$
\begin{gathered}
\int_{0}^{2 \pi} \cos m \theta \cos q \theta \mathrm{d} \theta=\pi\left(1+\delta_{m 0}\right) \delta_{m q}, \\
\int_{0}^{2 \pi} \sin m \theta \sin q \theta \mathrm{d} \theta=\pi\left(1-\delta_{m 0}\right) \delta_{m q} \\
\int_{0}^{2 \pi} \cos m \theta \sin q \theta \mathrm{d} \theta=0, \quad \text { and } \\
\int_{0}^{1} R_{n m}(\rho) R_{p q}(\rho) \rho \mathrm{d} \rho=\frac{\delta_{n p} \delta_{m q}}{2(n+1)} .
\end{gathered}
$$

Equations (10a) and (10b) are the main results of this paper. Please note that this definition of the beam quality factor is the square of the better established $M^{2}$, is expressed this way for convenience, and will be retained for the rest of the paper. The equations show that the beam quality factor is affected by individual amounts of $y$-astigmatism, $x$ - and $y$-coma, $x$ - and $y$ triangular astigmatism, spherical aberration, and the truncation parameter. It is generally established that tip/tilt and defocus do not affect the quality of the beam, and this is consistent with the above-mentioned results. One can understand this from the perspective that defocus is nothing but a curvature on the wavefront due to an ideal lens, whereas tip/tilt simply adjusts the propagation axis of the beam, a factor that is removed through the first moment subtraction of the field in deducing the second moment sizes. Interestingly, the $x$ astigmatism does not influence the beam quality factor, while $x$-astigmatism behaves very much like a normal lens (without the rotational symmetry associated with defocus) since the beam quality factor is calculated in the principal axes. With $y$-astigmatism, the lenslike behavior does not take place in the principal axes where the beam quality factor is calculated and thus has a deleterious impact on the laser beam quality. Note that, when dealing with astigmatic beams, the choice of principal axes is always an important factor in how the beam propagation is described $[12,13]$. In this work we have adopted the sensible approach of choosing the principal axes

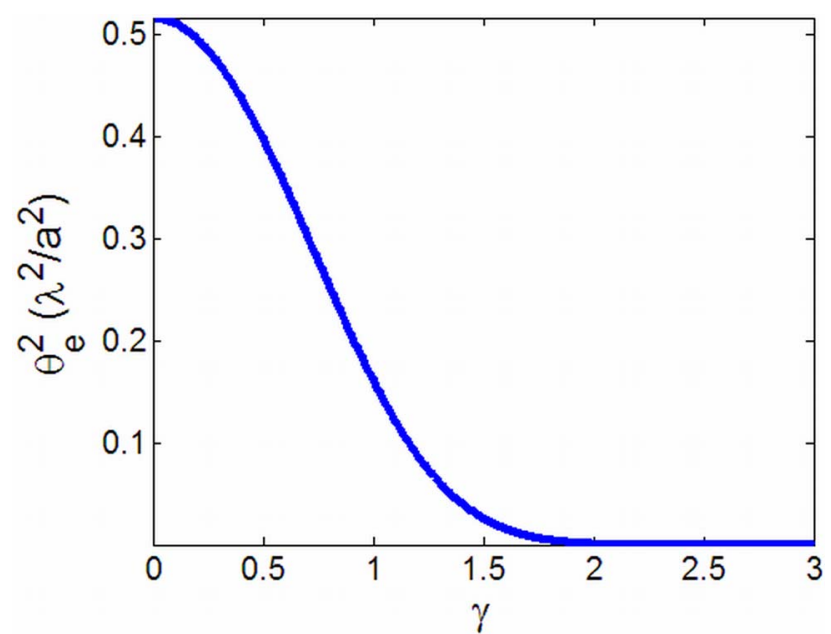

Fig. 1. (Color online) Added far-field divergence due to the aperture size as a result of truncation. 
Table 2. Special Cases of the Beam Quality Factor Equations

\begin{tabular}{ccc}
\hline & Beam Quality Factor, $M^{4}$ \\
\hline$\gamma \geq 2$ & $M_{x}^{4}$ & $1+24 \pi^{2} \gamma^{-8}\left(B_{22}^{2} \gamma^{4}+3\left(5 A_{31}^{2}+2 A_{31} A_{33}+A_{33}^{2}+\left(B_{31}^{2}-B_{33}^{2}\right)\right) \gamma^{2}+60 A_{40}^{2}\right)$ \\
& $M_{y}^{4}$ & $1+24 \pi^{2} \gamma^{-8}\left(B_{22}^{2} \gamma^{4}+3\left(5 B_{31}^{2}-2 B_{31} B_{33}+B_{33}^{2}+\left(A_{31}^{2}+A_{33}^{2}\right)\right) \gamma^{2}+60 A_{40}^{2}\right)$ \\
$\gamma \sim 0$ & $M_{x}^{4}$ & $16 / \pi+8\left(\pi^{2}\left(3 B_{22}^{2}+6\left(3 A_{31}^{2}+2 A_{31} A_{33}+A_{33}^{2}+\left(B_{31}+B_{33}\right)^{2}\right)+20 A_{40}^{2}\right)\right)$ \\
& $M_{y}^{4}$ & $16 / \pi+8\left(\pi^{2}\left(3 B_{22}^{2}+6\left(3 B_{31}^{2}-2 B_{31} B_{33}+B_{33}^{2}+\left(A_{31}+A_{33}\right)^{2}\right)+20 A_{40}^{2}\right)\right)$ \\
No aberrations & $M^{4}$ & $1+(4 / \pi)\left(\exp \left(2 \gamma^{2}\right)-1\right)^{-2}\left((\pi-16) \gamma^{2}-8+\pi \gamma^{4}+\exp \left(2 \gamma^{2}\right)\left(8-\pi \gamma^{2}\right)\right)$ \\
\hline
\end{tabular}

of the beam to coincide with that of the $x$ and $y$ planes of the Zernike basis functions. If one were to rotate the choice of principal axes by $45^{\circ}$, then the role of $x$ - and $y$-astigmatism would interchange ( $x$-astigmatism would now affect the beam quality factor), and in general it is the component of the as- tigmatism at $45^{\circ}$ to our choice of principal axes that influences the beam quality factor [5].

For completeness we point out that the equations can be reduced to cover the soft aperture case $(\gamma \geq 2)$ and a truncated plane-wave $(\gamma \sim 0)$ case and are given in Table 2 . An average of
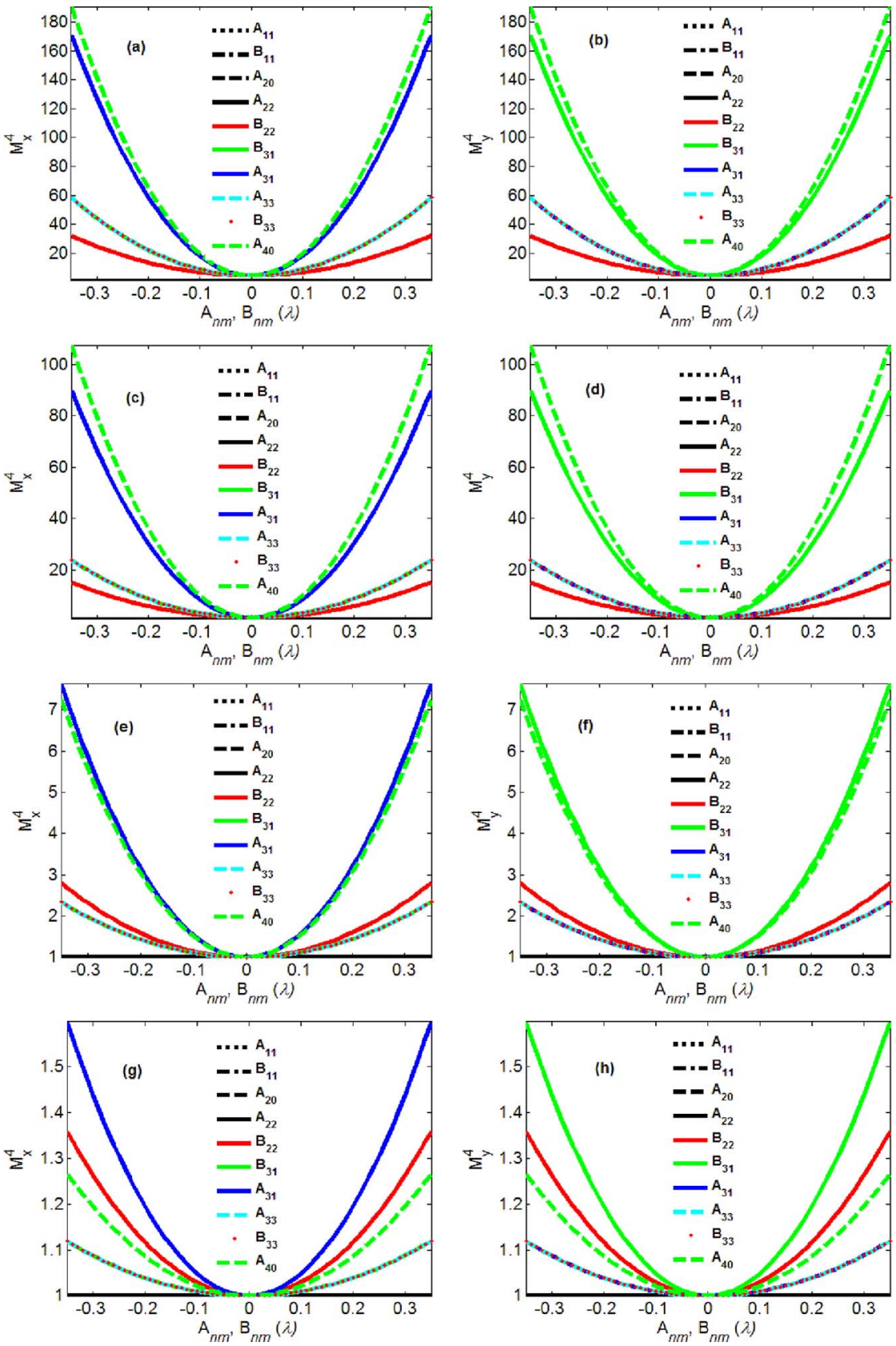

Fig. 2. (Color online) Beam quality factor of a truncated Gaussian beam for (a), (b) $\gamma=0.3$, (c), (d) $\gamma=1$, (e), (f) $\gamma=2$, and (g), (h) $\gamma=3$. 


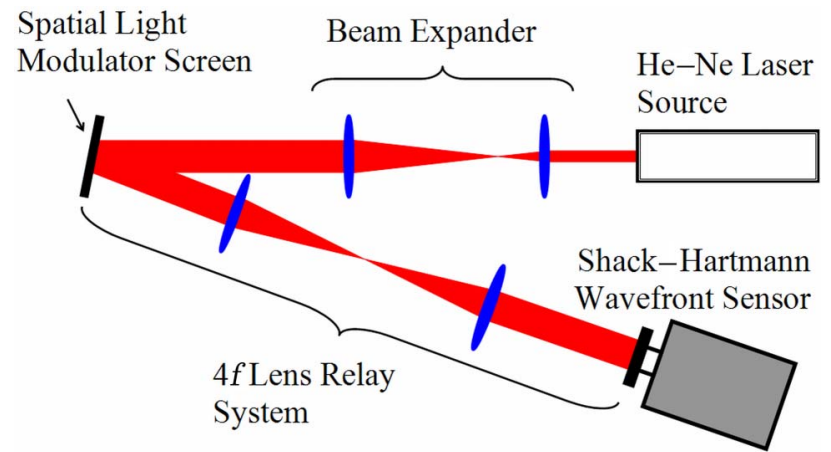

Fig. 3. (Color online) Experimental setup for the demonstration of optical aberrations in the laboratory. The digital holograms imparted to the laser beam are relay imaged to the wavefront sensor for an accurate phase measurement.

these two cases returns the previously reported result for the beam quality factor tensor, $J[14]$, for all aberrations except that of $y$-astigmatism ([14] reports no effect of $y$-astigmatism on $J$ ). The impact of the aperture alone can be deduced by setting all aberration coefficients to zero (see Table 2 ).

\section{NUMERICAL RESULTS IN THE CASE OF AN ABERRATED GAUSSIAN BEAM-HARD APERTURE}

To illustrate the usefulness of the theory, we calculate the impact of aberrations on the laser beam quality factor for the case of an aberrated and truncated Gaussian beam, as described by Eq. (10). As expected, there is no dependence for $x$-tilt $\left(A_{11}\right), y$-tilt $\left(B_{11}\right)$, defocus $\left(A_{20}\right)$, and $x$-astigmatism $\left(A_{22}\right)$, for which $M_{x}^{4}=M_{y}^{4}=1$ for all $\gamma$. All other aberrations show a monotonically increasing function for beam quality factor versus aberration coefficient, implying that no "balancing" of the aberrations is possible-i.e., one cannot improve the laser beam quality factor of an aberrated laser beam by introducing other types of aberrations. The model correctly accounts for the impact of the aperture size on the beam quality factor: increasing $\gamma$ (softer aperture) reduces the beam quality factor, as expected. We also note that, for small values of $\gamma$, the beam quality deterioration is mostly a result of spherical aberration closely followed by coma: $x$-coma in the horizontal plane and $y$-coma in the vertical plane. We note that, as $\gamma$ increases from 1 to 2, coma, in both axes, begins to dominate with the change over occurring at $\gamma=1.91$. The domination increases as $\gamma$ increases until $\gamma=3$, when $y$-astigmatism becomes the second most dominant [Figs. $\underline{2(\mathrm{~g})}$ and $\underline{2(\mathrm{~h})}$ ]. Last, we note that $B_{31}$, $A_{33}$, and $B_{33}$ have the same value of $M_{x}^{4}$ and that $A_{31}, A_{33}$ and $B_{33}$ have the same value of $M_{y}^{4}$, for all $\gamma$.

\section{EXPERIMENT SETUP AND RESULTS}

The experimental setup is shown in Fig. 3. A laser beam from an He-Ne source (Thorlabs, Model HRP020) was passed through a $4 \times$ beam expander onto a liquid crystal phase-only spatial light modulator (Holoeye, Model HEO1080P) onto which aberration coefficient holograms of 8 bit gray scale ranging from white ( 0 rad phase change) to black ( $2 \pi \mathrm{rad}$ phase change) were programmed in a $1920 \times 1080$ pixel array. The resulting aberrated beam was then imaged onto the detector grid of a Shack-Hartmann wavefront sensor (Wavefront Sciences, Model CLAS 2D) with a $4 f$ lens relay system, thus preserving both the amplitude and phase of the aberrated beam. Both the amplitude and phase of the aberrated laser beam could then be determined, as well as the resulting $M^{2}$ parameter.

Primary aberrations with selected coefficients were programmed onto the spatial light modulator (SLM). The modular- $2 \pi$ holograms of all primary aberrations are shown in Table 3 as gray scale images of size $1920 \times 1080$ pixels to match the SLM dimensions. The Zernike radius was set at $a=$ $1.44 \mathrm{~mm}$ to be one sixth of the SLM screen width in the smallest axis, with the laser beam width expanded to $\omega=1.9 \mathrm{~mm}$ $(\gamma=0.763)$.

A calibration process was carried out to make sure that the Zernike polynomials programmed onto the sensor were accurate to within a reasonable error margin [17]. For each aberration the selected coefficients were between $-0.3 \lambda$ and $0.3 \lambda$, and 20 frames of data were gathered for each aberration to allow averaging of the results.

The experimental results are presented in Fig. 4 for: (a) defocus, (b) spherical aberration, (c) $x$ - and (d) $y$-astigmatism, (e) $x$ - and (f) $y$-coma, and (g) $x$ - and (h) $y$-triangular astigmatism, with the theoretical prediction shown as a solid curve. The data are plotted as mean values with error bars of 1 standard deviation and given for both principal axes. Clearly the model, as given by Eq. (10), is in excellent agreement with the measured data. As expected, defocus does not impact on the beam quality factor, while spherical aberration adversely affects the quality of the laser beam following a simple power law: $M^{4} \propto A_{40}^{2}$, as found by others [12]. The $x$ astigmatism does not influence the beam quality factor, while

Table 3. Modular-2 $\pi$ Phase Screens of the Zernike Primary Aberrations

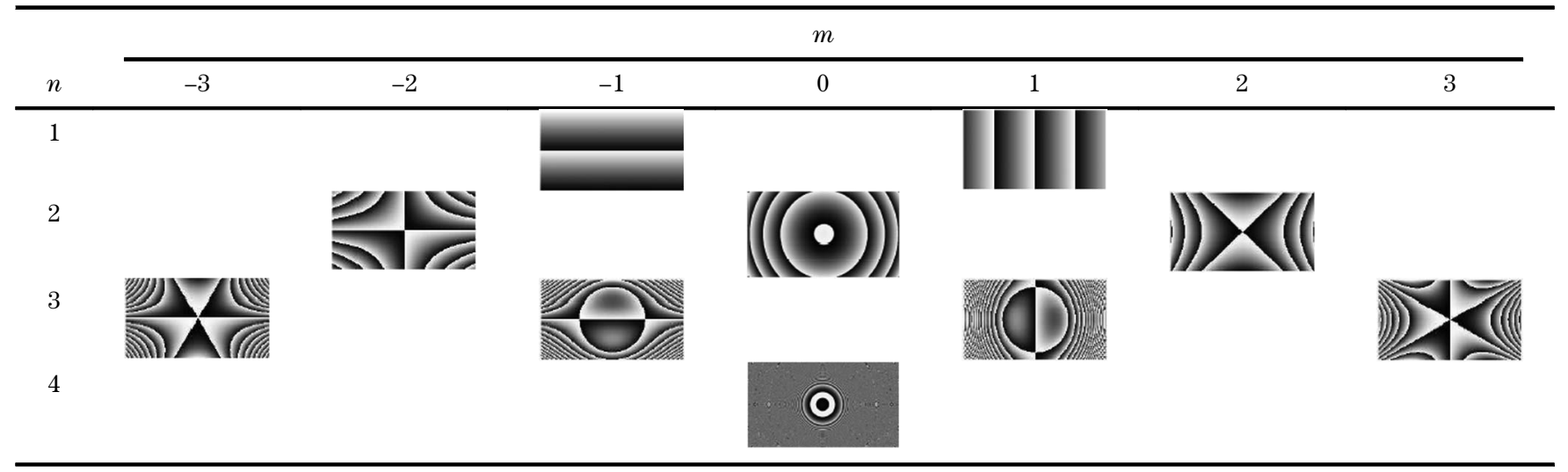



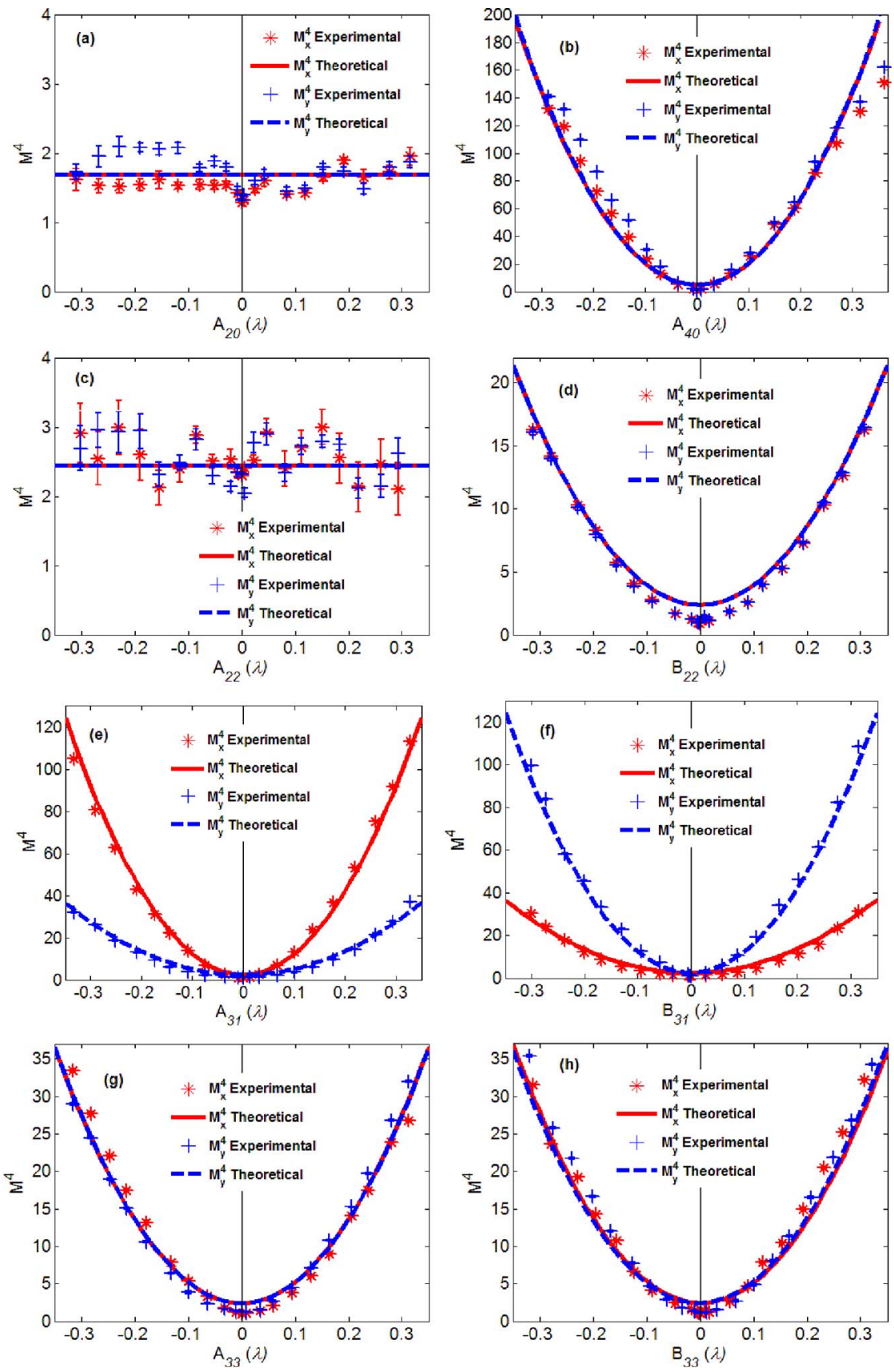

Fig. 4. (Color online) Beam quality factor dependence on rms Zernike primary aberrations (except tilt) in the $x$ axis and $y$ axis for the model (graphs) and the experimental results (data points).

the $y$-astigmatism does, thus confirming the departure of this model over that reported previously [14]. As expected, due to the asymmetry in coma, the resulting change in the beam quality factor is not the same in the two principal axes [see Figs. $\underline{4(\mathrm{e})}$ and $\underline{4(\mathrm{f})}$ ].

\section{CONCLUSION}

We have derived closed-form equations that relate the impact of an individual aberration on the beam quality factor. The result, which takes into account an arbitrary-sized aperture, has been verified experimentally using digitally encoded optical aberrations. These results should prove useful for practical optical design given the ease with which the beam quality factor may be calculated, as well as the accuracy of the model.

\section{APPENDIX A: DERIVATION OF EQ. (2)}

We outline here the derivation of Eq. (2) using the method of moments to derive an expression for the beam quality factor using the curvature removal method. We apply this method to a general complex amplitude transverse electric field, at some arbitrary propagation distance, given by

$$
U(x, y)=\psi(x, y) \exp (i \phi(x, y))
$$


where we have normalized the Cartesian plane $\left(x^{\prime}, y^{\prime}\right)$ to some aperture of size $a$ such that $x=x^{\prime} / a$ and $y=y^{\prime} / a$. The power carried by the field is then given by $P=a^{2} \iint \psi^{2}(x, y) \mathrm{d} x \mathrm{~d} y$. As the propagation for the two principal axes can be treated as separable problems, we outline the approach for the $x$ axis only, with the $y$ axis following an identical treatment. We make use of the standard definition of the beam quality factor as a product of moments [16]:

$$
M_{x}^{2}=\frac{4 \pi}{\lambda} \sqrt{\left\langle x^{2}\right\rangle_{0}\left\langle D_{x}^{2}\right\rangle}
$$

where each moment is defined by

$$
\left\langle x^{s} D_{x}^{t}\right\rangle=\frac{a^{s+2}}{P} \iint \psi^{2}(x-\langle x\rangle)^{s}\left(D_{x}-\left\langle D_{x}\right\rangle\right)^{t} \mathrm{~d} x \mathrm{~d} y,
$$

for arbitrary nonzero integers $s$ and $t$. Without any loss of generality, we may take the beam center to coincide with the aperture center so that $\langle x\rangle=0$. Note that, in this expression, the subscript 0 in the second-order spatial moment $(s=2$ and $t=0$ ) implies that the calculation is done at the waist plane:

$$
\left\langle x^{2}\right\rangle=\frac{a^{4}}{P} \iint \psi^{2} x^{2} \mathrm{~d} x \mathrm{~d} y .
$$

In the paraxial regime, the angle $D_{x}$ between the Poynting vector and the $z$ axis is given by

$$
D_{x}=\frac{\lambda}{2 \pi \psi a}\left|\frac{\partial U}{\partial x}\right|=\frac{\lambda}{2 \pi \psi a} \sqrt{\left(\frac{\partial \psi}{\partial x}\right)^{2}+\left(\psi \frac{\partial \phi}{\partial x}\right)^{2}} .
$$

Making the substitution $s=0$ and $t=2$ into Eq. (A3) and then substituting Eq. (A5) into the result we get

$$
\begin{aligned}
\left\langle D_{x}^{2}\right\rangle= & a^{2} \frac{1}{P} \iint \psi^{2}\left(D_{x}-\left\langle D_{x}\right\rangle\right)^{2} \mathrm{~d} x \mathrm{~d} y \\
= & \frac{\lambda^{2}}{4 \pi^{2}}\left(\frac{a^{2}}{P} \iint\left(\left(\frac{\partial \psi}{\partial x}\right)^{2}+\left(\psi \frac{\partial \phi}{\partial x}\right)^{2}\right) \mathrm{d} x \mathrm{~d} y\right. \\
& \left.-\left(\frac{a}{P} \iint \psi^{2} \frac{\partial \phi}{\partial x} \mathrm{~d} x \mathrm{~d} y\right)^{2}\right) .
\end{aligned}
$$

Now we note that the above approach can in fact be used for any propagation plane, not necessarily the waist plane, if the expression in Eq. (A2) is adjusted to create a "virtual" waist plane by subtracting the first-order spatial-angular moment $\left(\left\langle x D_{x}\right\rangle\right)$ from the second-order moments. This term is zero at the waist but is nonzero at any other plane. The new expression for the beam quality factor then becomes

$$
M_{x}^{2}=\frac{4 \pi}{\lambda} \sqrt{\left\langle x^{2}\right\rangle\left\langle D_{x}^{2}\right\rangle-\left\langle x D_{x}\right\rangle^{2}}
$$

where the first-order spatial-angular moment $(s=t=1)$ is given by

$$
\left\langle x D_{x}\right\rangle=\frac{a^{3}}{P} \iint \psi^{2} x D_{x} \mathrm{~d} x \mathrm{~d} y=\frac{\lambda}{2 \pi} \frac{a^{2}}{P} \iint \psi^{2} x \frac{\partial \phi}{\partial x} \mathrm{~d} x \mathrm{~d} y .
$$

The above expressions, together with Eq. (A7), allow the beam quality factor to be calculated at any plane if the amplitude and phase of the field at that plane are known. These expressions can also be formulated in terms of conventional beam size $\left(\omega_{x}=2\langle x\rangle\right)$, divergence $\left(\theta_{x}=2\left\langle D_{x}\right\rangle\right)$, and the firstorder spatial-angular moment $V_{x}=\omega_{x} \theta_{x}=4\left\langle x D_{x}\right\rangle$. The transformation to cylindrical coordinates is achieved through a simple coordinate change.

\section{REFERENCES}

1. A. Siegman, "New developments in laser resonators," Proc. SPIE 1224, 2-14 (1990).

2. T. F. Johnston, Jr., " $M^{2}$ concept characterizes beam quality," Laser Focus World 26, 173-184 (May 1990).

3. V. N. Mahajan, Optical Imaging and Aberrations, Part I: Ray Geometrical Optics (SPIE, 1998).

4. V. N. Mahajan, Optical Imaging and Aberrations, Part 2: Wave Diffraction Optics (SPIE, 1998).

5. G-M. Dai, Wavefront Optics for Vision Correction (SPIE, 2008).

6. M. Born and E. Wolf, Principles of Optics: Electromagnetic Theory of Propagation, Interference and Diffraction of Light, 7th ed. (Cambridge University, 1998), pp. 517-553.

7. R. J. Noll, "Zernike polynomials and atmospheric turbulence," J. Opt. Soc. Am. 66, 207-211 (1976).

8. A. Wada, H. Ohminato, T. Yonemura, Y. Miyamoto, and M. Takeda, "Effect of comatic aberration on the propagation characteristics of the Laguerre-Gaussian beams," Opt. Rev. 12, 451-455 (2005).

9. O. Mendoza-Yero and J. Alda, "Irradiance map of an apertured Gaussian beam affected by coma," Opt. Commun. 271, 517-523 (2007).

10. R. K. Singh, P. Senthilkumaran, and K. Singh, "The effect of astigmatism on the diffraction of a vortex carrying beam with a Gaussian background," J. Opt. A 9, 543-554 (2007).

11. R. Borghi and M. Santarsiero, " $M^{2}$ factor of Bessel-Gauss beams," Opt. Lett. 22, 262-264 (1997).

12. A. E. Siegman, "Analysis of laser beam quality degradation caused by quartic phase aberrations," Appl. Opt. 32 , 5893-5091 (1993).

13. J. A. Ruff and A. E. Siegman, "Measurement of beam quality degradation due to spherical aberration in a simple lens," Opt. Quantum Electron. 26, 629-632 (1994).

14. J. Alda, J. Alonso, and E. Bernabeu, "Characterization of aberrated laser beams,” J. Opt. Soc. Am. A 14, 2737-2747 (1997).

15. T. A. Jeong and J. Lee, "Accurate determination of the beam quality factor of an aberrated high-power laser pulse," J. Korean Phys. Soc. 55, 488-494 (2009).

16. R. Martinez-Herrero and P. M. Meijas, "Second-order spatial characterization of hard-edge diffracted beams," Opt. Lett. 18, 1669-1671 (1993).

17. C. Mafusire and A. Forbes, CSIR National Laser Centre, P.O. Box 395, Pretoria 0001, South Africa are preparing a manuscript to be called "Phase calibration of the Shack-Hartmann wavefront sensor using a phase only spatial sight modulator." 